\title{
Ethidium Bromide Resistance: A Selective Marker Located on Linkage Group IV of Dictyostelium discoideum
}

\author{
By MALCOLM D. WRIGHT, KEITH L. WILLIAMS* \\ AND PETER C. NEWELL \\ Department of Biochemistry, University of Oxford, South Parks Road, \\ Oxford $O X \mathrm{I} 3 Q U$
}

(Received 2 May 1977)

\section{INTRODUCTION}

The cellular slime mould Dictyostelium discoideum is a simple eukaryote that is eminently suitable for studies on differentiation and in which a parasexual system of genetic analysis is currently being developed. In this system two growth temperature-sensitive haploid strains are fused and diploids are selected at the restrictive temperature at a frequency of about $10^{-5}$ (Loomis, 1969; Katz \& Sussman, 1972). Recently an alternative diploid selection system using Bacillus subtilis sensitivity has been reported (Newell et al., 1977). The diploids so produced revert at low frequency to haploids through a process of transient aneuploidy (Sinha \& Ashworth, 1969; Brody \& Williams, 1974). Linkage analysis is possible since chromosome loss during haploidization is random and the frequency of mitotic crossingover is low (Mosses, Williams \& Newell, 1975). For parasexual genetics to be practicable in $D$. discoideum some means of selection is needed to isolate haploids and mitotic cross-over diploids from parental diploids (Katz \& Sussman, 1972). Distal selective markers are required on each chromosome, all of which probably have terminal centromeres (Robson \& Williams, 1977). So far selective markers have been reported on linkage groups I ( $c y c A$ and acr B, Katz \& Sussman, 1972; Williams, Kessin \& Newell, 1974a), II (acrA, Williams et al., I974a) and III ( $a c r C$, Rothman \& Alexander, 1975). These loci all involve recessive resistance to noxious compounds. In this report resistance to the intercalating dye ethidium bromide is described and the locus (designated $e b r A$ ) is assigned to linkage group IV.

\section{METHODS}

Chemicals. Acriflavin (neutral), ethidium bromide and cycloheximide were obtained from Sigma. Other chemicals, of the purest grade available, were purchased from BDH or Fisons.

Media and growth conditions. Amoebae or spores of D. discoideum were diluted in sterile SS salt solution (Sussman, 1966). The nutrient SM agar plates (Mosses et al., 1975) were prepared with Oxoid agar no. 3 ( $5 \mathrm{~g}^{-1}$ ), Difco Bacto-agar ( $5 \mathrm{~g} \mathrm{l}^{-1}$ ) or Calbiochem agar (I $\mathrm{g} \mathrm{l}^{-1}$ ).

Initially, ethidium bromide was added to SM Difco agar, after autoclaving, from a filter-sterilized stock solution. Latterly, unsterilized ethidium bromide has been added as a solid, or as a stock solution ( $3 \mathrm{mg}$ $\mathrm{ml}^{-1}$ ) to the agar after autoclaving. Media containing different brands of agar appeared to affect the toxicity of ethidium bromide. For this reason the final concentration of ethidium bromide used was $35 \mu \mathrm{g} \mathrm{m} \mathbf{l}^{-1}$ in SM Difco agar and $30 \mu \mathrm{g} \mathrm{ml}^{-1}$ in SM Calbiochem agar. (Medium containing Oxoid agar no. 3 appeared to be considerably less toxic and was not used for these studies.) As far as possible, the ethidium bromide plates were used under conditions of low light intensity, i.e. exposure to sunlight or germicidal lamps was avoided.

SM agar plates containing methanol $(2 \%, \mathrm{v} / \mathrm{v})$, acriflavin $\left(100 \mu \mathrm{g} \mathrm{m}^{-1}\right)$ or cycloheximide $\left(500 \mu \mathrm{g} \mathrm{ml}^{-1}\right)$ were prepared as described previously (Mosses et al., 1975). All agar plates were stored at $4{ }^{\circ} \mathrm{C}$ in the dark until required.

* Present address: Genetics Department, Research School of Biological Sciences, The Australian National University, Canberra City, A.C.T. Australia. 
Strains. The previously described haploid strain NPI4 is a cycloheximide- and methanol-resistant, whitespored and growth temperature-sensitive mutant containing the alleles cycAI, acrAI, whiA, tsgDI2. Strain $\mathrm{X2}$ is a brown pigmented and growth temperature-sensitive mutant containing the alleles bwnA and tsgAr (Williams et al., 1974a). Strain NP8I is a spontaneous ethidium bromide-resistant mutant of X2 containing the allele ebrAr. Strain X22 is a methanol-resistant, white- and round-spored, growth temperature-sensitive mutant containing the alleles acrAI, whiA, sprAI, tsgEr 3 and tsg Dr2 (Williams \& Newell, 1976), and strain HU26 is a spontaneous mutant of X22 resistant to cobaltous chloride and carries the allele cob-353. All strains were maintained as spores collected in horse serum, dried on to a silica gel and stored at $4{ }^{\circ} \mathrm{C}$. During experiments, clones were maintained on SM agar plates with the bacterium Klebsiella aerogenes.

Parasexual genetic techniques. Heterozygous diploid strains were constructed between haploids NPI4 and NP8I (diploid DP219) and between haploids HU26 and NP8I (diploid DU133). In each case the haploid strains carried non-allelic growth temperature-sensitive mutations and diploids were selected at the restrictive temperature as described previously (Williams, Kessin \& Newell, 1974b). Haploid segregants were obtained from these diploids by plating approximately $10^{4}$ amoebae or spores on to SM agar containing either cycloheximide, methanol or ethidium bromide. These plates were spread at the same time with $0.1 \mathrm{ml}$ of a thick suspension of $K$. aerogenes. In some experiments only haploids of certain independent origin were chosen (see Mosses et al., 1975). Haploids and diploids were distinguished by spore size.

\section{RESULTS AND DISCUSSION}

\section{Isolation of ethidium bromide-resistant mutants}

Spontaneous mutants resistant to ethidium bromide were isolated in strain X2 at a frequency of $10^{-6}$ by plating on SM agar containing ethidium bromide at $25 \mu \mathrm{g} \mathrm{ml}^{-1}$. Of several such mutants isolated, strain NP8I containing the mutation $e b r A I$ was chosen for further study. Strains containing the $e b r A 1$ mutation plated with about $50 \%$ efficiency on SM agar and SM agar containing ethidium bromide at up to $50 \mu \mathrm{g} \mathrm{ml}^{-1}$, although at this concentration growth was considerably slower than on SM agar. SM agar containing 30 or $35 \mu \mathrm{g}$ ethidium bromide $\mathrm{ml}^{-1}$ was generally used as this produced good growth on ethidium bromide and avoided leakiness in sensitive strains. At 30 to $35 \mu \mathrm{g}$ ethidium bromide $\mathrm{ml}^{-1}$, resistant clones appeared after 5 days compared with 3 days on SM agar.

\section{Dominance and assignment to a linkage group}

The ebrAI allele is recessive to wild type since diploids heterozygous for this marker, e.g. DP219, DU133, are sensitive to ethidium bromide but segregate resistant haploid and diploid clones at a frequency (haploids at $\mathrm{I} \times 10^{-4}$; diploids at $1.5 \times 10^{-4}$ ) similar to that found for other recessive resistance markers, e.g. cycA, acr $A$ (Williams et al., I974a; Mosses et al., 1975).

The $e b r A I$ locus has been located on linkage group IV by analysing haploid segregants of diploids DP2 I 9 and DUI 33 (Table I). Both diploids allowed examination of linkage groups I to IV, with DP2I9 allowing selection for linkage groups I (cycA), II (acrA) and IV (ebrA) and DUI 33 allowing selection for linkage groups II (acr $A$ ) and IV (ebrA). Of 947 haploid segregants examined 946 co-segregated ethidium bromide resistance with brown pigment (bwnA) on linkage group IV. Linkage groups I, II and III segregated independently of ethidium bromide resistance in both diploids (Table I), consistent with ebrAI being on linkage group IV. In DUI 33 ethidium bromide resistance segregated independently of $c o b-353$ which has recently been located on linkage group VII (Williams \& Newell, 1976; D. I. Ratner \& P. C. Newell, unpublished observations; K. L. Williams, unpublished observations).

\section{Deviations from the I: I segregation of unlinked markers}

The different segregant classes were recovered with unequal frequencies (Table I). When linkage group II was selected by plating on SM agar containing methanol, an excess of the haploid segregants were sensitive to ethidium bromide. In experiment $\mathrm{I}$, there were 78 $e b r A^{+}$haploids and $9 e b r A$. Similarly, in experiment 5, 110 of the 126 haploids selected on 
Table I. Assignment of ethidium bromide resistance (ebrAI) to linkage group IV by haploidization of diploids DP2 19 and DUI 33

Linkage group I was selected by plating clones on cycloheximide plates; linkage group II was selected on methanol plates; linkage group IV was selected on ethidium bromide plates. Temperature resistance was determined by replica-plating at $22{ }^{\circ} \mathrm{C}$ and at $27^{\circ} \mathrm{C}$. Experiments $\mathrm{I}$ to 6 involved 20, 20, 32, 16, 21 and 27 independent clones respectively.

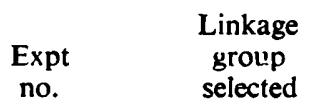

Diploid DP2 I9

I

2

3

4

Diploid DUI 33

5

6

\section{I}

II

IV

$$
\text { I }
$$

II

IV

IV

IV

II

IV
Ratio of haploid segregants in linkage group

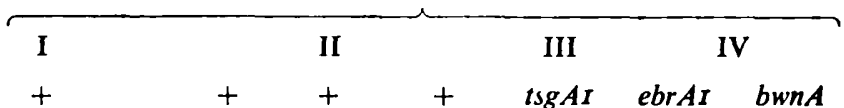

\begin{tabular}{|c|c|c|}
\hline+ & + & + \\
\hline whiA & $\operatorname{acr} A \boldsymbol{I}$ & $t s g D r 2$ \\
\hline
\end{tabular}

$\frac{34}{7}$

$\frac{0}{87}$

$\frac{47}{58}$

$\frac{8}{9}$

$\frac{0}{30}$

$\frac{8}{10}$

$\frac{t s g A I}{t} \quad \frac{e b r A I \quad b w n A}{+}$

$\frac{2}{37} \quad \frac{1}{40} \quad \frac{I}{40}$

ND

$\frac{9}{78} \quad \frac{9}{78}$

$\frac{43}{\text { II }} \quad \frac{105}{0} \quad \frac{105}{0}$

ND $\frac{15}{2} \quad \frac{15}{2}$

ND $\frac{20}{10} \quad \frac{19^{*}}{11}$

ND $\quad \frac{18}{0} \quad \frac{18}{0}$

$\frac{154}{43} \quad \frac{341}{0} \quad \frac{341}{0}$

$\frac{42}{20} \quad \frac{109}{0} \quad \frac{109}{0}$

$\frac{25}{84}$

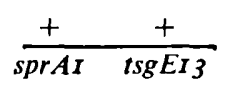

$\begin{array}{ccc}+ & + & + \\ \text { whiA acrAI } & + \text { tsgDI2 }\end{array}$

$\frac{t s g A I}{+}$

$\frac{e b r A I \quad b w n A}{t \quad t}$

$\frac{0}{126}$

ND $\quad \frac{16}{110} \quad \frac{16}{110}$

$\frac{37}{36}$

ND, Not determined.

* Includes an $e b r A I \quad b w n A^{+}$segregant which presumably arose by a cross-over prior to haploidization.

methanol carried $e b r A^{+}$. Ethidium bromide-resistant colonies grew more slowly than ethidium bromide-sensitive colonies on all types of plates (clones taking 5 rather than 3 days to achieve a given size).

When linkage group IV was selected by plating on SM agar containing ethidium bromide, both genotypes of linkage group II segregated equally (experiment $3 ; \mathrm{I} 77$ whi+ $\mathrm{i}^{+} 64$ whi) and all the colonies grew slowly. These results suggest that the $e b r A$ mutation, or a mutation(s) linked to $e b r A$ in strain NP8I, causes slow growth and possibly a lower plating efficiency than the wild-type allele(s). Hence when $e b r A I$ is not selected, the chromosome carrying this mutation is found at a lower frequency than the wild-type chromosome. This differential recovery must be borne in mind when using $e b r A$ with other selectors for linkage analysis.

Resistance to ethidium bromide establishes the first selective marker on linkage group IV. This marker should be of considerable use for assigning markers to linkage groups and for mitotic mapping within linkage group IV. 
M.D.W. was in receipt of a S.R.C. postgraduate studentship during the course of this work. P.C.N. thanks the S.R.C. for financial support.

\section{REFERENCES}

Brody, T. \& Williams, K. L. (1974). Cytological analysis of the parasexual cycle in Dictyostelium discoideum. Journal of General Microbiology 82, 37I-383.

Katz, E. R. \& Sussman, M. (1972). Parasexual recombination in Dictyostelium discoideum: selection of stable diploid heterozygotes and stable haploid segregants. Proceedings of the National Academy of Sciences of the United States of America 69, 495-498.

LoOMIs, W.F. (1969). Temperature-sensitive mutants of Dictyostelium discoideum. Journal of Bacteriology 99, 65-69.

Mosses, D., Williams, K. L. \& Newell, P. C. (1975). The use of mitotic crossing-over for genetic analysis in Dictyostelium discoideum: mapping of linkage group II. Journal of General Microbiology 90, 247-259.

Newell, P. C., Henderson, R. F., Mosses, D. G. \& Ratner, D. I. (1977). Sensitivity to Bacillus subtilis: a novel system for selection of heterozygous diploids of Dictyostelium discoideum. Journal of General Microbiology roo, 207-2 I I.

Robson, G. E. \& Williams, K. L. (1977). The mitotic chromosomes of the cellular slime mould Dictyostelium discoideum: a karyotype based on
Giemsa banding. Journal of General Microbiology 99, $19 \mathrm{I}-200$.

Rothman, F. G. \& Alexander, E. T. (1975). Parasexual genetic analysis of the slime mould Dictyostelium discoideum A3. Genetics 80, 71 5-731. SinHA, U. \& AsHWORTH, J. M. (I969). Evidence for the existence of elements of a parasexual cycle in the cellular slime mould Dictyostelium discoideum. Proceedings of the Royal Society Br73, 531-540.

Sussman, M. (1966). Biochemical and genetic methods in the study of cellular slime mould development. Methods in Cell Physiology 2, 397-410.

Williams, K. L. \& Newell, P. C. (1976). A genetic study of aggregation in the cellular slime mould Dictyostelium discoideum using complementation analysis. Genetics 82, 287-307.

Williams, K. L., Kessin, R. H. \& Newell, P. C. (1974a). Parasexual genetics in Dictyostelium discoideum: mitotic analysis of acriflavin resistance and growth in axenic medium. Journal of General Microbiology 84, 59-69.

Williams, K. L., Kessin, R. H. \& Newfll, P. C. $(1974 \mathrm{~b})$. Genetics of growth in axenic medium of the cellular slime mould Dictyostelium discoideum. Nature, London 247, 142-143. 